\title{
Comment on "Cost-Effectiveness of Cannabidiol Adjunct Therapy Versus Usual Care for the Treatment of Seizures in Lennox-Gastaut Syndrome"
}

\author{
Kelly Hollenack ${ }^{1}\left[\right.$ ] Jade Marshall ${ }^{2} \mathbb{D}$
}

Accepted: 6 February 2021 / Published online: 6 March 2021

(c) The Author(s) 2021

\section{Dear Editor,}

We read with interest three articles recently published in your journal on evaluating the cost effectiveness of add-on highly purified cannabidiol (CBD) to usual care for the treatment of patients with Lennox-Gastaut syndrome (LGS) and/ or Dravet syndrome (DS) in different markets [1-3]. CBD is approved as Epidiolex ${ }^{\circledR}$ in the USA (Greenwich Biosciences, Inc.) for the treatment of seizures associated with LGS, DS, or tuberous sclerosis complex in patients $\geq 1$ year of age [4].

Two of these articles found CBD to be a cost-effective treatment for LGS and/or DS, with incremental costeffectiveness ratios (ICERs) of Canadian dollars $\$ 32,399$ / quality-adjusted life year (QALY) [1], and $£ 33,721 / \mathrm{QALY}$ and $£ 32,471 / Q A L Y$ (model submitted to National Institute for Health Care and Excellence [NICE]) [2]. In addition, a previous analysis reported an ICER of \$14,688/QALY [5]. Conversely, the third article suggests that CBD would not be cost effective for LGS with an ICER of \$451,800/QALY [3]. As the original developer of the NICE model, we wish to enrich the ongoing discussion on the cost effectiveness of CBD for patients with LGS and DS.

In complex conditions such as LGS and DS, authors can make differing assumptions and employ alternative approaches to economic models leading to different conclusions. For example, Neuberger et al.'s model includes

This comment refers to the article available online at https://doi. org/10.1007/s40273-020-00945-z. Replies to this comment are available at https://doi.org/10.1007/s40273-021-01006-9 and https ://doi.org/10.1007/s40273-021-01007-8.

Kelly Hollenack

KHollenack@greenwichbiosciences.com

1 Greenwich Biosciences, Inc., 5750 Fleet Street, Suite 200, Carlsbad, CA 92008, USA

2 GW Pharma Ltd, London, UK health effects only in relation to patients, whereas the NICE model and Elliott et al.'s model both incorporate CBD's impact on reducing caregivers' burden. Patients with LGS and DS experience seizures associated with a risk of injury and mortality, have multiple comorbidities, and often require constant care [6-10]. The inclusion of caregivers was a key driver of the NICE model outcomes, with a greater QALY gain across multiple caregivers than the patient [2]. While there is no absolute right or wrong approach, we believe there are a number of factors that should be considered when comparing across studies.

First, the model structure employed must be justified based on suitability for the disease area, and evaluation parameters and should be consistent with the current clinical understanding of the disease [11]. Both Neuberger et al.'s and Elliott et al.'s models define health states based on the percentage reduction in seizures from baseline- the model structure used to evaluate other treatments in the early literature [12]. Patients are grouped based on relative (i.e., percentage point) seizure reduction, irrespective of baseline seizure frequency. A patient with a 50\% response would have the same cost and QALY assigned regardless of whether they started with 100 or 10 seizures. Additionally, the use of a $\leq 25 \%$ response to define a "non-responder" may overlook the fact that any reduction in seizures may be meaningful to the families of patients with LGS or DS [13], especially for patients with high baseline seizure counts. The NICE model implemented an alternative structure based on absolute seizure frequency and seizure-free days, the latter of which is considered a particularly meaningful outcome to patients as days with no seizures are likely to improve both the patient's quality of life (QoL) and ability to perform daily tasks [14]. Given the high seizure frequency associated with LGS and DS, model structures based on absolute seizure frequency and seizure-free days may be more appropriate to reflect the heterogeneity of the patient population and capture the 
expected cost and QoL benefits of an intervention in these highly refractory patient syndromes.

Second, because of the lack of comprehensive utility studies in the published literature, Neuberger et al.'s and Elliott et al.'s models use QoL values from an abstract that utilized vignettes describing percentage reductions in seizures [15]. However, with limited details of the methodology, the validity of these vignettes is unknown. Furthermore, the results suggest only a $7 \%$ improvement in utility from a $25-50 \%$ seizure reduction, despite a $50 \%$ seizure reduction being regarded as meaningful in both clinical trials and clinical practice [16, 17]. Considering these limitations and the need for more robust utility data to meet the alternative health state structure, utility data were generated for the NICE model based on direct measures of QoL [18]. Both fewer seizures and additional seizure-free days improved QoL in patients with LGS or DS and caregivers, with seizure-free days having the greatest impact (GW Research Ltd, data on file).

Third, different assumptions were made around the treatment regimen, leading to the divergence in results. Based on the described Weibull distribution, we believe Neuberger et al.'s model assumes that around $80 \%$ of patients would remain on therapy from age 15 years to death. Open-label clinical trial data for CBD indicates that $65 \%$ of patients with LGS and $45 \%$ with DS remain on treatment at 3 years (GW Research Ltd, data on file), which is unsurprising given these populations are known to cycle through multiple drugs [16, 19-21]. Neuberger et al.'s model also assumes that all patients discontinue therapy at the same rate, regardless of seizure response. In contrast, the NICE model enriches the study population for responders by assuming that those without an adequate response are more likely to discontinue, based on clinical trial data [22, 23].

Finally, as the original developer of the model submitted to NICE, we had the additional benefit of access to individual patient data from clinical trials. This offers a significant advantage in accurate modeling of non-linear treatment effects, rather than having to rely on average treatment effects based on summary data of the overall population.

In summary, we are grateful that Pharmacoeconomics provides an open platform for discussion. We recognize that appropriately modeling the value of CBD is not a simple task. Moreover, as the Second Panel on Cost Effectiveness in Health and Medicine notes, "QALYs may not accurately reflect the burden of short-lived but intense experiences. Thus the benefits of interventions that reduce the incidence of such experiences may be undervalued." [24]. We appreciate the approaches employed by Neuberger et al. and Elliott et al., which enrich ongoing discussion of the value of CBD and add to our growing knowledge of LGS and DS. We appreciate that both Neuberger et al. and Elliott et al. acknowledge the limitations of their approaches, including the lack of clinical validation, the validity of utility data, and how seizure reductions were incorporated, all of which may explain why the results have differed.

Acknowledgements Editorial support was provided to the authors by Jennifer Stewart, MSc, of Helios Medical Communications, Macclesfield, UK, and funded by GW Research Ltd.

Authors' Contribution The first draft of the letter was written by Kelly Hollenack and Jade Marshall. Both authors commented on previous versions of the letter and approved the final letter.

\section{Declarations}

Funding This work was sponsored by GW Research Ltd, Cambridge, UK.

Conflict of interest Kelly Hollenack is an employee of Greenwich Biosciences, Inc., Carlsbad, CA, USA, and Jade Marshall is an employee of GW Pharma Ltd, London, UK.

Ethics approval Not applicable.

Consent to participate Not applicable.

Consent for publication Not applicable.

Availability of data and material Not applicable.

Code availability Not applicable.

Open Access This article is licensed under a Creative Commons Attribution-NonCommercial 4.0 International License, which permits any non-commercial use, sharing, adaptation, distribution and reproduction in any medium or format, as long as you give appropriate credit to the original author(s) and the source, provide a link to the Creative Commons licence, and indicate if changes were made. The images or other third party material in this article are included in the article's Creative Commons licence, unless indicated otherwise in a credit line to the material. If material is not included in the article's Creative Commons licence and your intended use is not permitted by statutory regulation or exceeds the permitted use, you will need to obtain permission directly from the copyright holder. To view a copy of this licence, visit http://creativecommons.org/licenses/by-nc/4.0/.

\section{References}

1. Elliott J, McCoy B, Clifford T, Potter BK, Wells GA, Coyle D. Economic evaluation of cannabinoid oil for Dravet syndrome: a cost-utility analysis. Pharmacoeconomics. 2020;38:971-80.

2. Wijnen B, Armstrong N, Ramaekers B, Witlox W, Westwood M, Fayter D, et al. Cannabidiol for adjuvant treatment of seizures associated with Lennox-Gastaut syndrome and Dravet syndrome: an evidence review group perspective of a NICE single technology appraisal. Pharmacoeconomics. 2020;38:1043-53.

3. Neuberger EE, Carlson JJ, Veenstra DL. Cost-effectiveness of cannabidiol adjunct therapy versus usual care for the treatment of seizures in Lennox-Gastaut syndrome. Pharmacoeconomics. 2020;38:1237-45. 
4. US Food and Drug Administration. Epidiolex ${ }^{\circledR}$ (cannabidiol) oral solution: highlights of prescribing information. 2020. https ://www.epidiolex.com/sites/default/files/pdfs/0820/EPX-03645 -0820_EPIDIOLEX_\%28cannabidiol\%29_USPI.pdf. Accessed 10 July 2020.

5. Neuberger E, Veenstra DL. Cost-utility of cannabidiol in addition to background therapy versus background therapy alone in Lennox-Gastaut syndrome [abstract PND20]. Value Health. 2019;22(Suppl. 2):S273.

6. Cross JH, Auvin S, Falip M, Striano P, Arzimanoglou A. Expert opinion on the management of Lennox-Gastaut syndrome: treatment algorithms and practical considerations. Front Neurol. 2017;8:505.

7. Gibson PA. Lennox-Gastaut syndrome: impact on the caregivers and families of patients. J Multidiscip Healthc. 2014;7:441-8.

8. Wirrell EC, Nabbout R. Recent advances in the drug treatment of Dravet syndrome. CNS Drugs. 2019;33:867-81.

9. Campbell JD, Whittington MD, Kim CH, VanderVeen GR, Knupp $\mathrm{KG}$, Gammaitoni A. Assessing the impact of caring for a child with Dravet syndrome: results of a caregiver survey. Epilepsy Behav. 2018:80:152-6.

10. Mastrangelo M. Lennox-Gastaut syndrome: a state of the art review. Neuropediatrics. 2017;48:143-51.

11. Elliott J, van Katwyk S, McCoy B, Clifford T, Potter BK, Skidmore $\mathrm{B}$, et al. Decision models for assessing the cost effectiveness of treatments for pediatric drug-resistant epilepsy: a systematic review of economic evaluations. Pharmacoeconomics. 2019;37:1261-76.

12. Elliott J, McCoy B, Clifford T, Wells GA, Coyle D. Economic evaluation of stiripentol for Dravet syndrome: a cost-utility analysis. Pharmacoeconomics. 2018;36:1253-61.

13. Berg AT, Kaiser K, Dixon-Salazar T, Elliot A, McNamara N, Meskis MA, et al. Seizure burden in severe early-life epilepsy: perspectives from parents. Epilepsia. 2019;4:293-301.

14. Markowitz MA, Mauskopf JA, Halpern MT. Cost-effectiveness model of adjunctive lamotrigine for the treatment of epilepsy. Neurology. 1998;51:1026-33.

15. Yi Y, Verdian L, Oyee J, Tolley K, Heyes A. Eliciting utility scores for health states associated with Lennox-Gastaut syndrome [abstract PND22]. Value Health. 2008;11:A606.
16. Devinsky O, Patel AD, Cross JH, Villanueva V, Wirrell EC, Privitera $\mathrm{M}$, et al. Effect of cannabidiol on drop seizures in the LennoxGastaut syndrome. N Engl J Med. 2018;378:1888-97.

17. Sands TT, Rahdari S, Oldham MS, Caminha Nunes E, Tilton N, Cilio MR. Long-term safety, tolerability, and efficacy of cannabidiol in children with refractory epilepsy: results from an expanded access program in the US. CNS Drugs. 2019;33:47-60.

18. Radu X, Damera V, Martin M, Simontacchi K, Holland R. Quality of life in patients with Dravet syndrome or Lennox Gastaut syndrome in the UK: higher seizure frequency has a substantial negative impact on quality of life [abstract PR058]. Value Life. 2019;22(Suppl. 2):S346.

19. Devinsky O, Cross JH, Laux L, Marsh E, Miller I, Nabbout R, et al. Trial of cannabidiol for drug-resistant seizures in the Dravet syndrome. N Engl J Med. 2017;376:2011-20.

20. Thiele EA, Marsh ED, French JA, Mazurkiewicz-Beldzinska M, Benbadis SR, Joshi C, et al. Cannabidiol in patients with seizures associated with Lennox-Gastaut syndrome (GWPCARE4): a randomised, double-blind, placebo-controlled phase 3 trial. Lancet. 2018;391:1085-96.

21. Miller I, Scheffer IE, Gunning B, Sanchez-Carpintero R, GilNagel A, Perry MS, et al. Dose-ranging effect of adjunctive oral cannabidiol vs placebo on convulsive seizure frequency in Dravet syndrome: a randomized clinical trial. JAMA Neurol. 2020;77:613-21.

22. Devinsky O, Nabbout R, Miller I, Laux L, Zolnowska M, Wright $\mathrm{S}$, et al. Long-term cannabidiol treatment in patients with Dravet syndrome: an open-label extension trial. Epilepsia. 2019;60:294-302.

23. Thiele E, Marsh E, Mazurkiewicz-Beldzinska M, Halford JJ, Gunning B, Devinsky O, et al. Cannabidiol in patients with LennoxGastaut syndrome: interim analysis of an open-label extension study. Epilepsia. 2019;60:419-28.

24. Sanders GD, Neumann PJ, Basu A, Brock DW, Feeny D, Krahn $\mathrm{M}$, et al. Recommendations for conduct, methodological practices, and reporting of cost-effectiveness analyses: second panel on costeffectiveness in health and medicine. JAMA. 2016;316:1093-103. 